\title{
CALET GBM Observations of Gamma-ray Bursts and Gravitational Wave Sources
}

\author{
Kazutaka Yamaoka* for the CALET collaboration \\ Department of Particle Physics and Astronomy, Nagoya University, Furo-cho, Chikusa-ku, \\ Nagoya 464-8601 \\ E-mail: vamaokadisee.nagova-u.ac.jp
}

\begin{abstract}
The CALET Gamma-ray Burst Monitor (CGBM) is secondary scientific instrument of the Calorimetric Electron Telescope (CALET) mission on the International Space Station (ISS). The primary instrument Calorimeter (CAL) is capable of detecting gamma-ray bursts (GRBs) in the $\mathrm{GeV}-\mathrm{TeV}$ range, and the CGBM was attached to complement CAL gamma-ray observations in the keV-MeV range. The CGBM consists of 2 Hard X-ray Monitors (HXMs) and one Soft Gammaray Monitor (SGM), utilizing different scintillators $\mathrm{LaBr}_{3}(\mathrm{Ce})$ and $\mathrm{BGO}$ respectively. The CGBM covers a broadband energy range of $7 \mathrm{keV}-20 \mathrm{MeV}$ with a wide field of view (FOV). Since the launch on August 19, 2015, the CGBM has been sucessfully operated on the ISS for about 1.5 years, and detecting about 50 GRBs (roughly $20 \%$ short GRBs among them) per year as expected from pre-launch estimation. The CALET also concluded memolandum of understanding (MOU) with LIGO/Virgo collaboration, and we are searching for hard X-ray and gamma-ray counterparts for gravitational wave (GW) sources. In this paper, we will report on CGBM in-orbit operation, performance and observations of GRBs and GW sources.
\end{abstract}

35th International Cosmic Ray Conference - ICRC2017-

10-20 July, 2017

Bexco, Busan, Korea

${ }^{*}$ Speaker. 


\section{Introduction}

Gamma-ray bursts (GRBs) remain still exciting, but unsolved and mysterious objects even after 50 years of its discovery in 1960's. GRBs are classified into mainly two categories: short-hard and long-soft GRBs based on the duration-hardness diagram. These two categories can suggest at least two different origins: supernova explosions for long-soft GRBs and compact star mergers (neutron star (NS)-NS, NS-black hole (BH) etc.) for short-hard GRBs, although several other origins are proposed such as giant flares from soft gamma repeaters (SGRs) in a distant galaxy. For long-soft GRBs, it has been observationally confirmed that a part of them have been associated with supernovae explosions through optical measurements. But for short-hard GRBs, it can give only an indirect evidence that some of them are found in host galaxies with inactive star-formation regions. Hence, gravitational wave (GW) detection can be direct evidence to clarify the origin of GRBs. Since September 2015, GW events have been discovered by Laser Interferometer Gravitationalwave Observatory (LIGO). All the four events including one candidate observed so far are BH-BH mergers at $400-1000 \mathrm{Mpc}$, and there is no firm evidence for X-rays and gamma-ray counterparts since counters are not expected theoretically for BH-BH mergers. If the NS-NS and NS-BH mergers occur in nearby galaxies, we will expect to radiate both electromagnetic (EM) and GWs. Thus, a search for EM counterparts to GW sources are keys for finding GRB origins.

The Calorimetric Electron Telescope (CALET) is Japan-US-Italy collaborative mission dedicated for detecting high energy electrons and gamma-rays in the GeV-TeV range [四]. The main scientific objective is to clarify an origin of $\mathrm{TeV}$ electrons including possible contributions from nearby supernova remnants, and also to search for dark matter signals from undiscovered particles such as WIMPs (Weakly Interacting Massive Particles) expected in the GeV-TeV range. It was launched on August 19, 2015 by the H-IIB launch vehicles from JAXA Tanegashima Space Center and attached to the Exposed Facility of the Kibo experimental module on the International Space Station (ISS). The primary instrument for CALET is Calorimeter (CAL). In order to complement CAL gamma-ray observation functions, gamma-ray burst monitor (CGBM) was also attached as the secondary scientific instrument [[] [B]. By using two instruments, CAL and CGBM, we will be able to monitor transient X-ray and gamma-ray sky with a wide field of view (FOV). The CALET operation is fortunately matched to the beginning of the GW astronomy, so we will be able to contribute to GRB and GW science.

In this paper, we will focus on the secondary instrument CGBM and describe in-orbit operation and performance, and preliminary results about GRBs and GW sources from initial 1.8-year operations. Details of the CAL gamma-ray performance are given in separate papers [ [ 9 [ [ $]$ ].

\section{CALET Gamma-ray Burst Monitor (CGBM)}

The CGBM is similar type of scintillation detectors readout by photo-multiplier tubes to Fermi/GBM and CGRO/BATSE. It consists of two identical hard X-ray monitors (HXMs: referred as HXM1 and 2) and one soft gamma-ray monitor (SGM) to cover a wide energy range of $\sim 7 \mathrm{keV}$ to $\sim 20 \mathrm{MeV}$. The HXM utilizes thin $\operatorname{LaBr}_{3}(\mathrm{Ce})$ scintillator which has not been used for celestial observations in space yet, while the SGM utilizes thick BGO crystal with a high stopping power. Both monitors have a wide FOV for $\sim 3$ str. and $\sim 8$ str. for HXM and SGM respectively. To retain 
a wide energy coverage, we further installed amplifiers with two different gains (high and low) in the analog electronics, and their pulse heights were digitized by different Analog-to-Digital Converter (ADCs). Figure $\square$ shows CGBM on-axis effective area in comparison with those of other GRB instruments. Table $\square$ shows detector characteristics of CGBM.

In order to carry out efficient GRB observations with CALET, the CGBM has on-board trigger algorithm which detects a count rate increase above statistical background fluctuations in the electronics. When a rate increase is detected, i.e. a GRB on-board trigger happens, 1) a low energy threshold of the primary instrument CAL is changed from $\sim 10 \mathrm{GeV}$ to $\sim 1 \mathrm{GeV}$ (Low Energy Gamma-ray Mode) to detect GeV photons from GRBs. 2) event-by-event data with fine time resolution are available to allow a detailed temporal and spectral study of GRBs, and 3) two optical images with an exposure of $0.5 \mathrm{sec}$ are taken by the star sensor ASC (Advanced Stellar Compass) to detect optical flashes during the prompt emission phase.

The CGBM provides two types of scientific data (see Table $[$ ). One is histogram data regularly output as the telemetry. It consists of the time history (TH) data with $1 / 8 \mathrm{sec}$ and 8 energy channels, and pulse height (PH) data with $4 \mathrm{sec}$ and 512 channels. These data allows us a background monitoring in the ISS orbit and also a study of a period change in X-ray pulsars and as well as search for untriggered GRBs and transients. The other is event data which has an information for individual photons recorded with a high time resolution of $62.5 \mu \mathrm{s}$. This data is suitable for a detailed study of short GRBs. All the CGBM data will be made publicly available in this fall 2017.

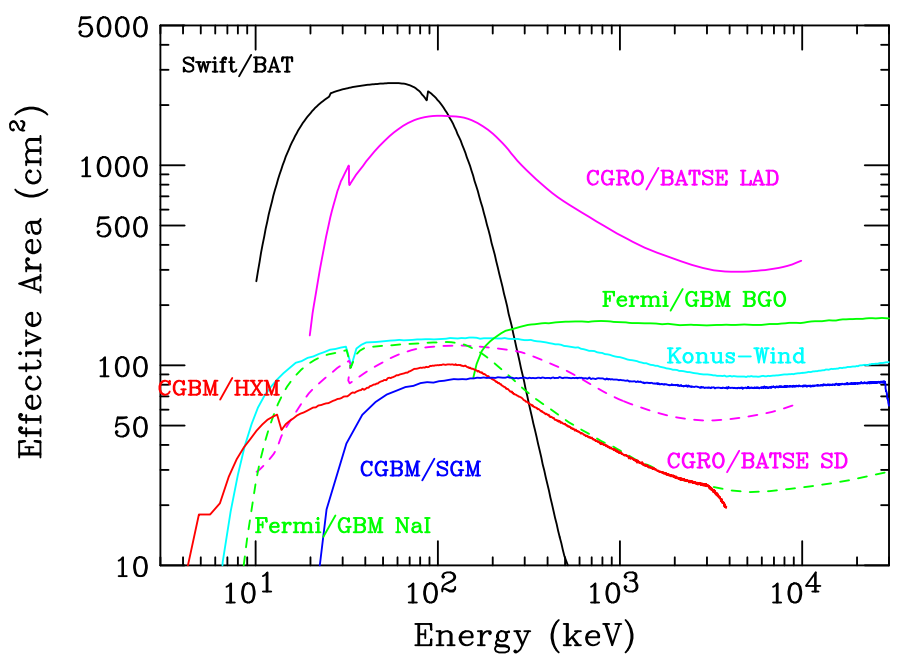

Figure 1: CGBM on-axis effective area as a function of energy in compared with other GRB instruments. The CGBM is relatively small detector, but sensitive to a wide energy range from $\sim 7 \mathrm{keV}$ to $\sim 20 \mathrm{MeV}$.

After attachment to the exposed facility (EF) of the Japanese Experimental Module (JEM) on August 25, 2015, the CGBM initial checkout started on September 22, 2015. Following three-days long-term running operation on October 5-7, nominal operation started on October 8, 2015. A high voltage (HV) on the PMTs is set at 700.7, 793.8, and 867.3 V for HXM1, HXM2 and SGM respectively. Currently we reduce the HVs at South Atlantic Anomaly (SAA) and high latitude regions to avoid PMT damages. This operation is done by both scheduled command and automatic 
Table 1: CGBM Detector Characteristics

\begin{tabular}{lcc}
\hline \hline Items & $\mathrm{HXM}$ & $\mathrm{SGM}$ \\
\hline Detector (Crystal) & $\mathrm{LaBr}_{3}(\mathrm{Ce})$ & $\mathrm{BGO}\left(\mathrm{Bi}_{3} \mathrm{Ge}_{4} \mathrm{O}_{12}\right)$ \\
Number & 2 & 1 \\
Diameter $(\mathrm{cm})$ & 6.1 & 10.2 \\
Thickness $(\mathrm{cm})$ & 1.27 & 7.6 \\
Energy Range $(\mathrm{keV})$ & $7-1000$ & $40-20000$ \\
Energy Resolution & $18 \% @ 32 \mathrm{keV}$ & $12 \% @ 662 \mathrm{keV}$ \\
Field of View (FOV) & $\sim 3 \mathrm{sr}$. & $\sim 8 \mathrm{sr}$. \\
\hline
\end{tabular}

Table 2: CGBM Data Types

\begin{tabular}{lccc}
\hline \hline Data & Time History (TH) & Pulse Height $(\mathrm{PH})$ & Event \\
\hline Type & \multicolumn{1}{c}{ Histogram } & Event-by-event \\
Time Resolution & $1 / 8 \mathrm{~s}$ & $4 \mathrm{~s}$ & $62.5 \mu \mathrm{s}$ \\
Energy Channels & 4(High) / 4 (low) & $102($ High) $/ 410($ Low $)$ & 4096 (High, Low) \\
Availability & Always output as the telemetry & Only available when a trigger happens. \\
\hline
\end{tabular}

operation using the in-orbit geographical map for protection. The observation efficiency is $\sim 58 \%$ per day.

The threshold level in the GRB trigger system has been set at $8.5 \sigma$ in the $10-50 \mathrm{keV}$ range (HXM) and 7.0бin the 40-230 keV range (SGM) after several changes. This setting is roughly estimated to a sensitivity for a few photons $\mathrm{cm}^{-2} \mathrm{~s}^{-1}$ in the $10-1000 \mathrm{keV}$ range. All the sensors and electronics have been working nominally after the launch.

\section{Gamma-ray Bursts (GRBs)}

The broadband spectroscopy is crucial to understand mechanisms of GRB prompt emissions which are still debated among GRB community. The synchrotron shock model has been widely accepted for a long time, but is inconsistent with several observational facts in terms of high radiation efficiency and narrow SED distributions. Since Monitor of All-sky X-ray Image (MAXI) mission is at the same platform on the ISS, CALET-MAXI collaboration is a unique capability for studying GRBs from $2 \mathrm{keV}$ up to $\mathrm{GeV}$ gamma-rays.

As of end of May 2017, the CGBM has detected 65 GRBs, corresponding to a GRB detection rate of about 40 per year. 12 out of 65 GRBs, $18 \%$ of total GRBs, are classified into short GRB category. 43 and 5 GRBs were simultaneously detected by Fermi-GBM and MAXI. The CGBM also sends an alert with occurrence time to world-wide community as a GCN Notice. The detailed information has been distributed as GCN circulars.

We will introduce two interesting GRBs from CGBM GRB database: GRB160107A and GRB 160625B. The long GRB 160107A was detected by CGBM at 22:20:43.20 (UT) [四], while MAXI detects 47 seconds prior to the CGBM trigger (22:19:56 UT) [四]. The MAXI and CGBM light 


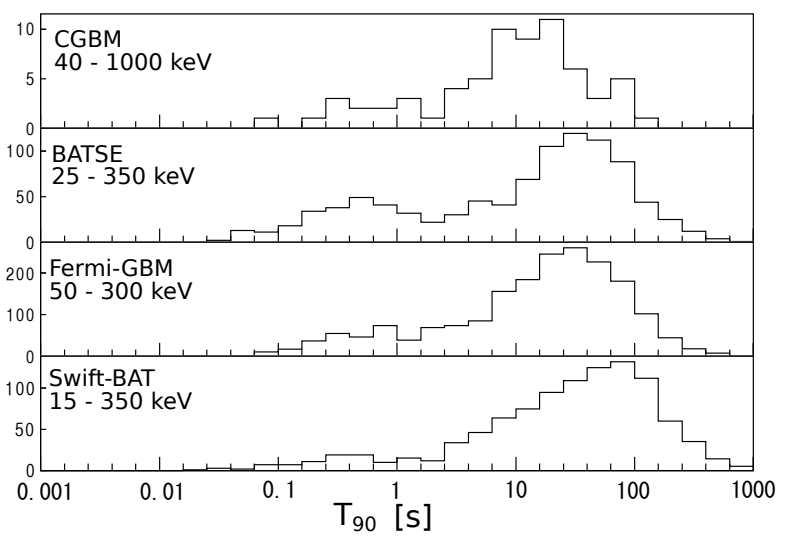

Figure 2: $\mathrm{T}_{90}$ duration distributions in compared with other GRB detectors. CGBM, Fermi-GBM, CGRO/BATSE, and Swift/BAT are shown from upper to lower.

curves are shown in Figure [3. Interestingly MAXI only see the initial soft part of GRBs in the 2-20 $\mathrm{keV}$ range, while CGBM can see very weak hard X-ray emission. This result suggests that such GRB beginning phase might be missed in current GRB monitors and its soft emission might come from photospheric emission. Detailed analysis will be reported in Kawakubo et al. in preparation.

The very bright, long GRB 160625B was detected by CGBM at 22:40:15.49 (UT) [8]. This GRB was detected by many other instruments such as Fermi-GBM and LAT, Konus-Wind, INTEGRAL SPI-ACS and MAXI (out of FOV). The Fermi-LAT detected about 300 photons above $100 \mathrm{MeV}$ [Q]. The fluence and peak flux were estimated to be $5.0 \times 10^{-4} \mathrm{ergs} \mathrm{cm}^{-2}$ and $167 \pm 1$ photons $\mathrm{cm}^{-2} \mathrm{~s}^{-1}$ in the $10-1000 \mathrm{keV}$ range. At the same time, the count rate of Charged Detector (CHD; $>\sim 600 \mathrm{keV}$ ) which is placed at the top of CAL increases significantly. Figure 1 shows CGBM HXM1, 2 and SGM spectra of this GRB. The photons were clearly detected up to $20 \mathrm{MeV}$. The direction to this GRB was interrupted by the ISS solar paddles, so the calibration and spectral analysis are still under way.

\section{Gravitational Wave (GW) sources}

A search for EM counterpart to GW sources is another important topic for CGBM. The current sky regions localized by LIGO are very large $\left(\sim 1000\right.$ degrees $\left.^{2}\right)$, so CALET instruments are suitable for search thanks to its wide FOVs. The CALET collaboration has signed Memorandum of Understanding (MoU) with LIGO-Virgo Collaborations (LVC) to follow-up GW events before the CALET launch. Under this agreement, the CALET collaboration has received prompt alert from LVC. The LIGO observations has been carried out twice during the first observation (O1: September 12, 2015 to January 19, 2016) and second (O2: November 30, 2016 to mid. 2017) runs. Three GW events have been significantly detected so far: GW 150914 [U]] and 151226 [ए]] (one candidate LVT 151012) in the O1 run and 170104 [ए2] in the O2 run. For the first GW 150914, unfortunately CALET was during the checkout phase, so neither of CAL nor CGBM were turned on. For other two events GW 151226 and 170104, trigger times were observable with both CAL 


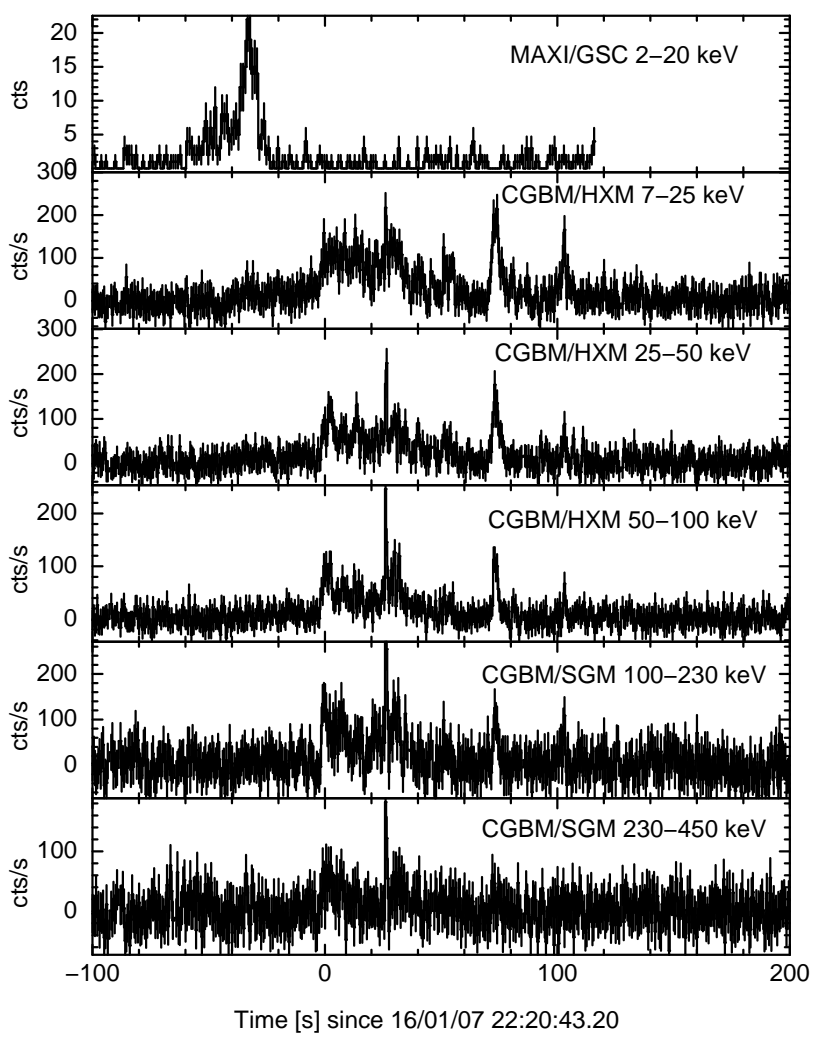

Figure 3: MAXI (upper panel) and CGBM HXM1+2 (middle) and SGM (lower) light curves of GRB 160107A. The MAXI visible window to GRB direction is shown by a red triangle. The MAXI detects soft $\mathrm{X}$-ray emissions at 47 seconds prior to the main episode detected by CGBM.

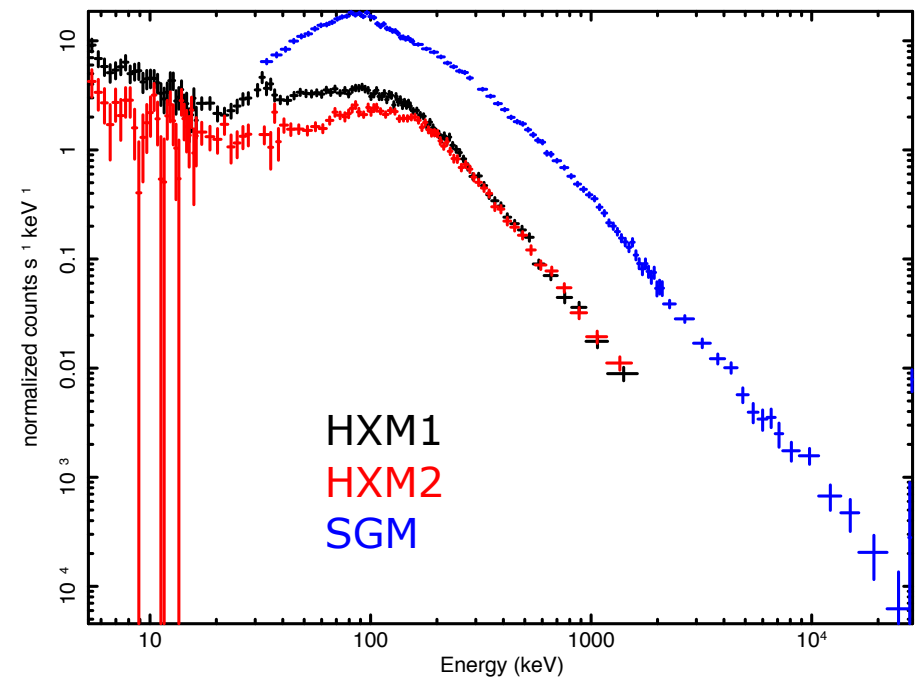

Figure 4: CGBM raw count spectra of GRB 160625B. X-ray and gamma-ray photons are clearly detected uo to $20 \mathrm{MeV}$. 
and CGBM. For GW 151226, CGBM covered 32\% (HXM) and 49\% (SGM) for sky locations, but could not see any transient events. The $7 \sigma$ upper limits for 1-s exposure are $1.0 \times 10^{-6} \mathrm{erg} \mathrm{cm}^{-2}$ $\mathrm{s}^{-1}$ in the $7-500 \mathrm{keV}(\mathrm{HXM})$, and $1.8 \times 10^{-6} \mathrm{~cm}^{-2} \mathrm{~s}^{-1}$ in the $50-1000 \mathrm{keV}$ range (SGM). Assuming the distance of $440 \mathrm{Mpc}$, the luminosity is estimated to be $3-5 \times 10^{49} \mathrm{erg} \mathrm{s}^{-1}$. This value is well below the typical luminosity for short GRBs $\left(\sim 10^{51} \mathrm{erg} \mathrm{s}^{-1}\right)$. It can be explained by either 1) there is little EM radiation as expected in the BH-BH mergers, or 2) collimated jet emission from mergers was not along our line of sight. or 3) it is located at outside of the CGBM FOV. These results were already published in Adriani et al. (2016) [[3]]. For GW 170104, we could detect $\mathrm{X}$-ray and gamma-ray counterpart with neither CAL nor CGBM. We give a similar upper limit for the flux to GW 151226 [14]]. We will also look for EM counterpart to high energy neutrino events detected by IceCube and ANTARES, but we could not find any counterparts up to present.

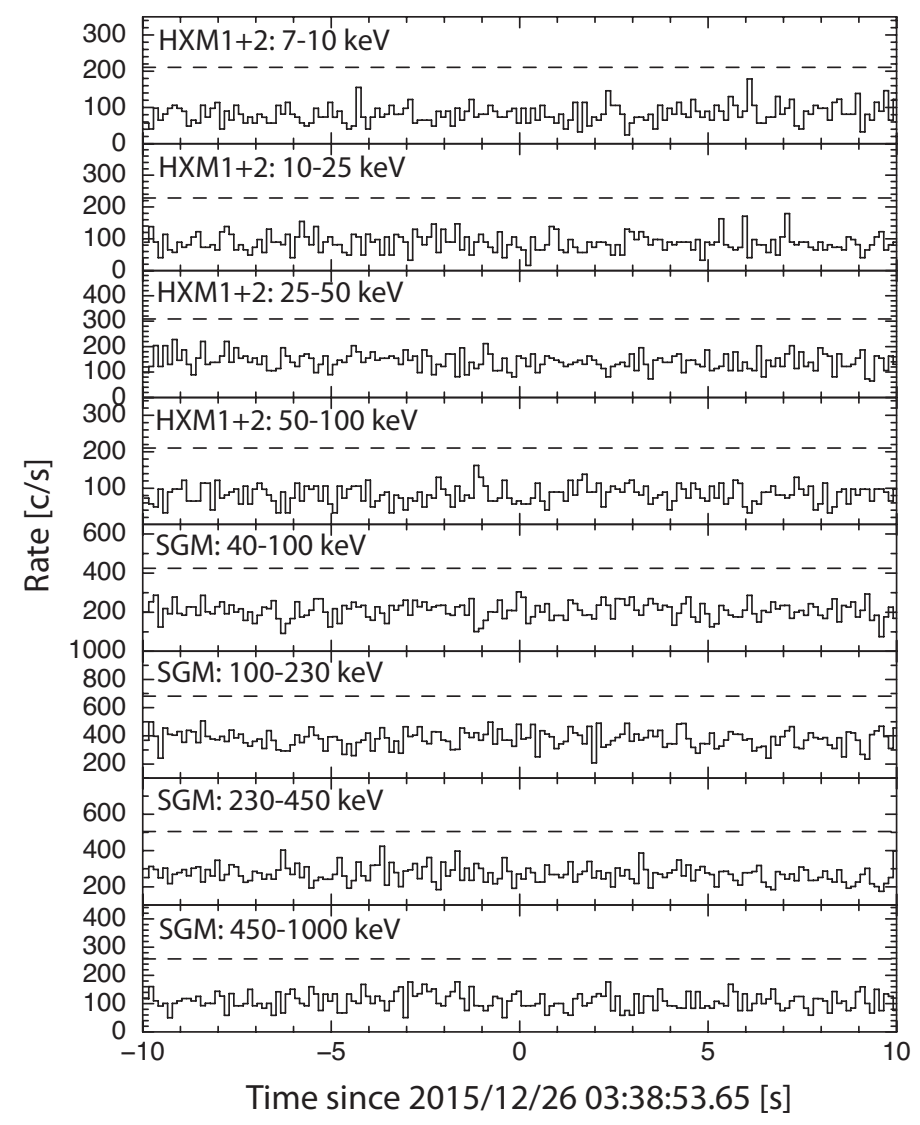

Figure 5: CGBM light curves with 1/8 sec time resolution around the trigger time (03:38:53.65 UT) of GW 151226. This figure is adopted from Adriani et al. (2016) [[33].

\section{Summary}

We have operated the CALET Gamma-ray Burst Monitor (CGBM) on the ISS for about 1.8 years since October 2015. The CGBM is detecting a rate of 40 GRBs per year (65 GRBs during 1.8 years operation), and 5 GRBs were detected by MAXI in the $2-20 \mathrm{keV}$. The CALET is fully 
Table 3: CALET status at trigger times of GW events.

\begin{tabular}{lcccccr}
\hline \hline GW events & $\begin{array}{c}\mathrm{T}_{0} \\
(\mathrm{UT})\end{array}$ & $\begin{array}{c}\text { SN } \\
\text { ratio }\end{array}$ & $\begin{array}{c}\text { Sky Loc. } \\
\left(\mathrm{deg}^{2}\right)\end{array}$ & $\begin{array}{c}\text { Status } \\
\text { CAL /CGBM }\end{array}$ & $\begin{array}{c}\text { Detection } \\
\text { CAL / CGBM }\end{array}$ & Ref. \\
\hline GW 150914 & $09: 50: 45$ & 24 & 230 & not operated / not operated & - & - \\
LVT 151012 & $09: 54: 43.44$ & 9.7 & 1600 & operated (HEG) / HV off & No / - & \\
GW 151226 & $03: 38: 53.65$ & 13 & 850 & operated (LEG) / operated & No / No & [ए3] \\
GW 170104 & $10: 11: 58.6$ & 13 & 1200 & operated (HEG) / operated & No / No & [ए4] $]$ \\
\hline
\end{tabular}

operating in the GW Astronomy era. A part of sky locations for two GW events GW 151226 and 170104 were within the CALET field of vies, but we could not find any X-ray and gamma-ray counterpart. We will continue finding EM counterparts to GW and neutrino events with CALET, and hope to detect them in a near future.

This work is partly supported by Grant-in-Aid (24684015 KY) from the Ministry of Education, Culture, Sports, Science, and Technology (MEXT).

\section{References}

[1] S. Torii et al., 2017, in this proceeding

[2] K. Yamaoka for the CALET Collaboration, Proc. 7th Huntsville Gamma-Ray Burst Symposium (Nashville, USA, 2013), paper 41 in eConf Proceedings C1304143.

[3] S. Nakahira for the CALET Team, Gamma-ray Burst Observations with MAXI and CALET, 7 years of MAXI: monitoring X-ray Transients, held 5-7 December 2016 at RIKEN.

[4] N. Cannady for the CALET Collaboration, 2017 in this proceedings.

[5] M. Mori for the CALET Collaboration, 2017, in this proceedings.

[6] S. Nakahira et al., 2016, GRB 160107A: CALET Gamma-Ray Burst Monitor detection, GCN Circ. 18845

[7] Y. E. Nakagawa et al., 2016, GRB 160107A: MAXI/GSC detection, GCN Circ. 18839

[8] S. Nakahira et al., 2016, GRB 160625B: CALET Gamma-Ray Burst Monitor detection, GCN Circ. 19617

[9] F. Dirirsa et al., 2016, GRB 160625B: Fermi-LAT refined analysis, GCN Circ. 19586

[10] B. P. Abbott et al., 2017, Observation of Gravitational Waves from a Binary Black Hole Merger, Phys. Rev. Lett. 116, 061102

[11] B. P. Abbott et al., 2017, GW151226: Observation of Gravitational Waves from a 22-Solar-Mass Binary Black Hole Coalescence, Phys. Rev. Lett. 116, 241103

[12] B. P. Abbott et al., 2017, GW170104: Observation of a 50-Solar-Mass Binary Black Hole Coalescence at Redshift 0.2, Phys. Rev. Lett. 118, 221101

[13] O. Adriani et al., Astrophys. J. Lett., 829: L20 (2016).

[14] T. Sakamoto et al., 2017, LIGO/Virgo G268556: CALET Observations, GCN Circ. 20339 